\title{
A Representation of the Virasoro Algebra Via Wigner-Heisenberg Algebraic Technique to Bosonic Systems
}

\author{
E. L. da Graça ${ }^{a, b}$, H. L. Carrion ${ }^{a}$, and R. de Lima Rodrigues ${ }^{a *}$ \\ ${ }^{a, b}$ Centro Brasileiro de Pesquisas Físicas \\ Rua Dr. Xavier Sigaud, 150, CEP 22290-180, Rio de Janeiro-RJ, Brazil \\ ${ }^{b}$ Departamento de Física Universidade Federal Rural do Rio de Janeiro \\ Antiga Rodovia Rio-São Paulo Km 47, BR 465, CEP 23.890-000, Seropédica-RJ
}

Received on 2 May, 2002. Revised version received on 12 December, 2002.

\begin{abstract}
Using the Wigner-Heisenberg algebra for bosonic systems in connection with oscillators we find a new representation for the Virasoro algebra.
\end{abstract}

\section{Introduction}

In 1950, Wigner[1] proposed the interesting question, "Do the equations of motion determine the quantum-mechanical commutation relations?" and found as answer a generalized quantum rule for the one-dimensional harmonic oscillator. In the next year, Yang [2] found the coordinate representation for the linear momentum operator. Yang's wave mechanical description was further studied by Ohnuki et al. [3] and Mukunda et al. [4]. Recently, the general Wigner-Heisenberg (WH) oscillator algebra $[5,6,7,8]$ has been investigated in the context of the deformed algebra [9]. There, the author shows that finite-dimensional representations of the deformed parafermionic algebra with internal $Z_{2}$-grading structure. The superealization of the $\mathrm{WH}$ algebra has been independently considered in two works $[8,10]$.

The Virasoro algebra has been several applications in literature, let us point, for stance, the connections with the conformal group [11], construction as $s u(1,1)$ extension [12], supervirasoro [13] and quantum algebras [14].

In this work, starting from the Wigner-Heisenberg algebraic technique for the bosonic systems in connection with general oscillator, we find a new representation for the Virasoro algebra.

This work is arranged in the following way. In Section II, we present the WH algebra. In Section III, a representation of the modified Virasoro algebra is found. The conclusions are drawn in the Section IV.

\section{The WH algebra}

The Wigner Hamiltonian expressed in the symmetrized bilinear form in terms of the mutually adjoint abstract opera- tors $\hat{a}^{ \pm}$, defined by

$$
\hat{H}_{W}=\frac{1}{2}\left(\hat{p}_{x}^{2}+\hat{x}^{2}\right)=\frac{1}{2}\left[\hat{a}^{-}, \hat{a}^{+}\right]_{+}=\frac{1}{2}\left(\hat{a}^{-} \hat{a}^{+}+\hat{a}^{+} \hat{a}^{-}\right),
$$

where

$$
\hat{a}^{ \pm}=\frac{1}{\sqrt{2}}\left( \pm i \hat{p}_{x}-\hat{x}\right)
$$

Wigner showed that Heisenberg's equations of motion

$$
\left[\hat{H}_{W}, \hat{a}^{ \pm}\right]_{-}= \pm \hat{a}^{ \pm},
$$

do not necessarily entail in the usual quantum rule

$$
\left[a^{-}, a^{+}\right]_{-}=1 \Rightarrow\left[\hat{x}, \hat{p}_{x}\right]_{-}=i, \quad \hbar=1,
$$

but a more general quantum rule $[2,3,4]$ given by

$$
\left[\hat{a}^{-}, \hat{a}^{+}\right]_{-}=1+c \hat{R} \Longrightarrow\left[\hat{x}, \hat{p}_{x}\right]_{-}=i(1+c \hat{R}),
$$

where $c$ is a real constant, related to the ground state en$\operatorname{ergy} E_{W}^{(0)} \geq 0$ by virtue of the positive semi-definite form of $\hat{H}_{W}{ }^{1}$

$$
|c|=2 E^{(0)}-1,
$$

which is called Wigner parameter.

The basic (anti-)commutation relations (1) and (3), together with the derived relation (5), are referred to as constituting the WH algebra. It is a parabose algebra [5] for the degree of freedom. The WH algebra is obtained by combining also the requeriment that $\hat{x}$ satisfies the classical equation of motion $(\ddot{\hat{x}}+\hat{x}=0)$.

\footnotetext{
*Permanent address: Departamento de Ciências Exatas e da Natureza, Centro de Formação de Professores, Universidade Federal de Campina Grande, Cajazeiras - PB, 58.900-000, Brazil.

${ }^{1}$ Note that the case $c=0$ corresponds to the usual oscillator with $E^{(0)}=\frac{1}{2}, \quad \hbar=\omega=1$.
} 

ties

Note that $\hat{R}$ is an abstract operator satisfying the proper-

$$
\begin{gathered}
{\left[\hat{R}, \hat{a}^{ \pm}\right]_{+}=0 \Rightarrow\left[\hat{R}, \hat{H}_{W}\right]_{-}=0 ;} \\
\hat{R}^{\dagger}=\hat{R}^{-1}=\hat{R}, \quad \hat{R}^{2}=1,
\end{gathered}
$$

where one has used the following notation for the (anti- ) commutation relations: $[A, B]_{+} \equiv A B+B A$ and $[A, B]_{-} \equiv A B-B A$. Besides, we have

$$
\begin{aligned}
H_{W} & =\hat{a}^{+} \hat{a}^{-}+\frac{1}{2}(1+c \hat{R}) \\
& =\hat{a}^{-} \hat{a}^{+}-\frac{1}{2}(1+c \hat{R}) .
\end{aligned}
$$

Abstractly (O'Raifeartaigh and Ryan [6], Boulware and Deser [7]) $\hat{R}$ is the Klein operator, $\pm \exp \left\{i \pi\left(\hat{H}_{W}-E_{W}^{(0)}\right)\right\}$.

In the mechanical representation first investigated by Yang[2], $\hat{R}$ is realized by the Parity operator $P$ :

$$
\begin{gathered}
P|x>=|-x>\Rightarrow P x P^{-1}=-x, \\
P p_{x} P^{-1}=-p_{x}, \quad P^{-1}=P, \quad P^{2}=1 .
\end{gathered}
$$

Indeed, Yang [2] found the coordinate representation for the momentum operator $p_{x}$ as given by

$$
\begin{gathered}
\hat{p}_{x} \longrightarrow p_{x}=-i \frac{d}{d x}+i \frac{c}{2 x} P, \quad \hat{x} \longrightarrow x, \\
\hat{a}^{ \pm} \longrightarrow a_{\frac{c}{2}}^{ \pm}=\frac{1}{\sqrt{2}}\left( \pm \frac{d}{d x} \mp \frac{c}{2 x} P-x\right) .
\end{gathered}
$$

Yang's wave mechanical description was further investigated in $[3,4]$.

\section{Modified Virasoro algebra}

The elements of the Virasoro algebra $[11,13]$ will be represented in terms of ladder operators $a^{ \pm}$of quantum oscillators with or without deformation.

\section{A. Canonical representation of the Virasoro al- gebra}

The conformal group, $G$, in 2 dimensions consists of all general transformations:

$$
\Gamma: z \rightarrow \xi(z), \quad \bar{\Gamma}: \bar{z} \rightarrow \bar{\xi}(\bar{z}),
$$

where $\Gamma$ is a group of the more general transformation with one coordinate, or equivalently is a group of the diffeomorphism transformations in one dimension. The same situation for $\bar{\Gamma}$. Then, $G$ is given by the direct product, viz.,

$$
G=\Gamma \otimes \bar{\Gamma} .
$$

In the literature, $\Gamma$ and $\bar{\Gamma}$ are usually referred to as chiral and anti-chiral components of the conformal algebra in 2-dimensions. The algebra associated to the $\Gamma$ is called the Witt algebra or the classical Virasoro algebra $\left(\mathcal{L}_{0}\right)$.

If $\mathcal{G}$ is the algebra associated to the group $G$, then:

$$
\mathcal{G}=\underbrace{\mathcal{L}_{0}}_{\text {Witt }} \oplus \underbrace{\mathcal{L}_{0}}_{\text {Witt }} .
$$

Infinitesimal transformations of the group $\Gamma$ are: $z \rightarrow$ $z+\varepsilon(z)$, where $\varepsilon(z)$ is an infinitesimal analytical function. It can be represented as an infinite Laurent series, viz.,

$$
\varepsilon(z)=\sum_{n} \varepsilon_{n} z^{n+1}, \quad n \epsilon \mathbf{z} .
$$

Therefore, the Lie algebra $\mathcal{L}_{0}$ of the $\Gamma$ coincides with the algebra of differential operators defined in $\mathbf{C}-\{\mathbf{0}\}$ :

$$
\ell_{n}=z^{n+1} \frac{d}{d z}, \quad n=0, \pm 1, \pm 2, \cdots .
$$

The commutation relations have the following form:

$$
\left[\ell_{n}, \ell_{m}\right]=(n-m) \ell_{n+m}
$$

We shall denote the algebra (17) as $\mathcal{L}_{0}$, which admits a unique 1-dimensional central extension:

$$
\mathcal{L}_{\kappa}=\mathcal{L}_{0} \oplus \kappa \text { (The Virasoro algebra), }
$$

with the following commutation relations

$$
\begin{aligned}
{\left[\ell_{n}, \kappa\right] } & =0 \\
{\left[\ell_{n}, \ell_{m}\right] } & =(n-m) \ell_{n+m}+\kappa \frac{m^{3}-m}{12} \delta_{n+m, 0},
\end{aligned}
$$

where the value of the central charge " $\kappa$ " is the parameter of the theory into the context of Quantum Field Theory. The generators $\ell_{-1}, \ell_{0}, \ell_{1}$ form the subalgebra $\operatorname{sl}(2, \mathbf{R}) \subset \mathcal{L}_{0}$.

In this subsection, we consider the oscillatory representation of the elements for the Virasoro algebra without modification. Using the canonical commutation relation of the quantum mechanics (4), we obtain the following commutator:

$$
\left[\left(a^{-}\right)^{n+1}, a^{+}\right]_{-}=(n+1)\left(a^{-}\right)^{n}, \quad n=0,1,2, \cdots .
$$

From this commutator, we see that the Virasoro operators for the unidimensional harmonic oscillator can be defined by

$$
L_{n}=\left\{\begin{array}{l}
\left(a^{-}\right)^{n+1} a^{+}, \\
a^{-}\left(a^{+}\right)^{n+1},
\end{array}\right.
$$

which satisfy the following Virasoro algebra:

$$
\left[L_{n}, L_{m}\right]_{-}=(n-m) L_{n+m},
$$

where $n \geq 0$ and $m \geq 0$. Next, we consider the oscillatory representation in terms of the Wigner oscillator.

\section{B. Deformed Virasoro algebra}

Let us now consider the modified Virasoro algebra in terms of new ladder operators which satisfy the generalized commutation relation given by Eq. (5). Indeed, considering $L_{n}=\left(a^{-}\right)^{n+1} a^{+}$, we see that the Eq. (20) becomes: 


$$
\left[\left(a^{-}\right)^{n+1}, a^{+}\right]_{-}=\left\{\begin{array}{c}
(n+1)\left(a^{-}\right)^{n}+c P\left(a^{-}\right)^{n}, \quad n=2 k \\
(n+1)\left(a^{-}\right)^{n}, \quad n=2 k+1
\end{array}\right.
$$

where $k=0,1,2,3 \cdots$. Now we investigate the three possible cases for the Virasoro algebra.

Case (i): Two even indexes.

In this case, the Virasoro algebra is not changed, i.e.

$$
\left[L_{2 n}, L_{2 m}\right]_{-}=2(n-m) L_{2 n+2 m} .
$$

Case (ii): Two odd indexes.

In this case, the Virasoro algebra is not also changed, i.e.

$$
\left[L_{2 n+1}, L_{2 m+1}\right]_{-}=2(n-m) L_{2 n+2 m+2} .
$$

Case (iii): One even index and one odd index and vice-verse. In this case, the Virasoro algebra is changed, i.e.,

$$
\left[L_{2 n}, L_{2 m+1}\right]_{-}=2(n-m) L_{2 n+2 m+1}-(1-c P) L_{2 n+2 m+1} .
$$

Note that we get an anomalous term containing the parity operator $P$. Besides, we can obtain the three possible cases for the Virasoro adjoint operators $L_{n}^{\dagger}=a^{-}\left(a^{+}\right)^{n+1}$.

The question we formulate now is the following: What is the behaviour of the Virasoro operator on the autokets of the Wigner oscillator quantum states? To answer this question, one must first note that the Wigner oscillator ladder operators on autokets of these quantum states are given by

$$
\begin{aligned}
a_{\frac{c}{2}}^{-} \mid 2 m, \frac{c}{2}> & =\sqrt{2 m} \mid 2 m-1, \frac{c}{2}> \\
a_{\frac{c}{2}}^{-} \mid 2 m+1, \frac{c}{2}> & =\sqrt{2\left(m+E^{(0)}\right)} \mid 2 m, \frac{c}{2}> \\
a_{\frac{c}{2}}^{+} \mid 2 m, \frac{c}{2}> & =\sqrt{2\left(m+E^{(0)}\right)} \mid 2 m+1, \frac{c}{2}> \\
a_{\frac{c}{2}}^{+} \mid 2 m+1, \frac{c}{2}> & =\sqrt{2(m+1)} \mid 2 m+2, \frac{c}{2}>.
\end{aligned}
$$

A detailed analysis on this question will appear in a forthcoming paper.

\section{Conclusion}

In this work, we analyze the Wigner-Heisenberg algebra to bosonic systems in connection with oscillators and, thus, we find a new representation for the Virasoro algebra. Acting the annihilation operator(creation operator) in the Fock basis $\mid 2 m+1, \frac{c}{2}>\left(\mid 2 m, \frac{c}{2}>\right)$ the eigenvalue of the ground state of the Wigner oscillator appears only in the excited states associated with the even(odd) quanta given by Eq.(27). We show that only in the case associated with one even index and one odd index in the operator $L_{n}$ the Virasoro algebra is changed.
The super-realization of the Wigner-Heisenberg algebra proposed by Jayaraman and Rodrigues [8], and independently by Plyushchay [10], has been changed to investigate a potential model that describes a hydrogen atom with parastatistics [15].

\section{Acknowledgments}

The authors are grateful to the J. A. Helayel Neto for the kind hospitality at CBPF-MCT and for fruitful discussions. RLR and HLC are grateful to the CNPq - Brazil for their post-doctoral and doctoral Graduate fellowships, respectively. The authors would also like to thank Plyushchay and Zachos for the kind interest in pointing out relevant references on the subject of this paper. This work was presented in the XXII Brazilian National Meeting on Particles and Fields (October/2001).

\section{References}

[1] E.P. Wigner, Phys. Rev. 77711 (1950).

[2] L.M. Yang, Phys. Rev. 84, 7881951.

[3] Y. Ohnuki and S. Kamefuchi, J. Math. Phys. 19, 67 (1978); Y. Ohnuki and S. Watanabe J. Math. Phys. 333653 (1992).

[4] J.K. Sharma, C.L. Mehta, N. Mukunda and E.C.G. Sudarshan, J. Math. Phys. 22, 78 (1981); N. Mukunda, E.C.G. Sudarshan, J.K. Sharma, and C.L. Mehta, J. Math. Phys. 21, 2386 (1980); J.K. Sharma, C.L. Mehta and E.C.G. Sudarshan, J. Math. Phys. 19, 2089 (1978).

[5] H.S. Green, Phys. Rev. 90, 270 (1953); A.J. Macfarlane J. Math. Phys. 35, 1054 (1994).

[6] L. O'Raifeartaigh and C. Ryan, Proc. R. Irish Acad. A62, 93 (1963).

[7] D.G. Boulware and S. Deser, Il Nuovo Cimento 230XXX 230 (1963).

[8] J. Jayaraman and R. de L. Rodrigues, J. Phys. A: Math. Gen. 23, 3123 (1990).

[9] M.S. Plyushchay, Nucl. Phys. B491, 619 (1997).

[10] M.S. Plyushchay, Int. J. of Mod. Phys. A15, 3679 (2000).

[11] F. Toppan, "Conformal Field Theories," preprint UCP-HEP90/7; A.A. Belavin, A.M. Polyakov, A.B. Zamoloochikov, Nucl. Phys. B241, 333 (1984).

[12] D.B. Fairlie and C.K. Zachos, Phys. Lett. B202, 320 (1988).

[13] D.B. Fairlie, J. Nuyts, and C.K. Zachos, Commun. Math. Phys. 117, 595 (1988).

[14] T.L. Curtright, K. Cosmas, C.K. Zachos, Phys. Lett. B243, 237 (1990).

[15] J. Beckers and N. Debergh, Phys. Lett. A178, 43 (1993). 